\title{
Histone deacetylase inhibitors alter the expression of molecular markers in breast cancer cells via microRNAs
}

\author{
YEHUI SHI ${ }^{1}$, YONGSHENG JIA ${ }^{1,2}$, WEIPENG ZHAO ${ }^{1,2}$, LIYAN ZHOU $^{3,4}$, \\ XIAOJUAN XIE ${ }^{3,4}$ and ZHONGSHENG TONG ${ }^{1,2}$ \\ ${ }^{1}$ Department of Breast Oncology; ${ }^{2}$ Tianjin Medical University Cancer Institute and Hospital, Tianjin Medical University, \\ Tianjin 300060; ${ }^{3}$ National Clinical Research Center for Cancer, Key Laboratory of Breast Cancer Prevention \\ and Therapy, Tianjin Medical University; ${ }^{4}$ National Clinical Research Center, Key Laboratory of \\ Cancer Prevention and Therapy, Tianjin Medical University, Tianjin 300060, P.R. China
}

Received September 12, 2016; Accepted January 25, 2018

DOI: $10.3892 /$ ijmm.2018.3616

\begin{abstract}
Histone deacetylase inhibitors (HDACis) are able to suppress breast cancer cells in vitro and in vivo by altering the expression of estrogen receptor (ER), progesterone receptor (PR) or human epidermal growth factor receptor 2 (Her $2 /$ neu). Since HDACis can alter the expression of various microRNAs (miRNAs/miRs), the present study aimed to examine the role of miRNAs in the effects of HDACis on breast cancer cells. We first examined the mRNA expression of ER, PR, and Her2/neu using RT-PCR and the protein levels of ER, PR, and Her2/neu using western blot analysis in MDA-MB-231 and BT474 cells, after trichostatin A (TSA) or vorinostat (SAHA) treatment. We then conducted miRNA expression profiling using microarrays after BT474 cells were treated with TSA or SAHA. Finally, we examined the effects of synthetic miR-762 and miR-642a-3p inhibitors on SAHA-induced downregulation of Her2/neu and SAHA-induced apoptosis and PARP cleavage in BT474 cells. The results indicated that TSA and SAHA dose-dependently enhanced the mRNA and protein expression levels of ER and PR in MDA-MB-231 and BT474 cells. In addition, the mRNA expression levels of Her2/neu were reduced in MDA-MB-231 cells, and the mRNA and protein expression levels of Her2/neu were reduced in BT474 cells in response to SAHA and TSA. Furthermore, treatment with TSA $(0.2 \mu \mathrm{M})$ or SAHA $(5.0 \mu \mathrm{M})$ induced a marked alteration in the expression of various miRNAs in BT474 cells. Notably, when cells were cotransfected with miR-762 and miR-642a-3p inhibitors, SAHA-induced downregulation of Her2/neu was inhibited, and SAHA-induced apoptosis and poly (ADP-ribose) polymerase cleavage were significantly reduced in BT474 cells. These results indicated that numerous HDACi-induced miRNAs are required to
\end{abstract}

Correspondence to: Dr Zhongsheng Tong, Department of Breast Oncology, Tianjin Medical University, 22 Qixiangtai Road, Heping, Tianjin 300060, P.R. China

E-mail: tongzhongsheng1@gmail.com

Key words: breast cancer, molecular marker, Her2/neu, microRNA, histone deacetylase inhibitors downregulate Her2/neu levels and promote apoptosis of Her2-overexpressing breast cancer cells.

\section{Introduction}

Breast cancers are often classified by stage, pathology, grade, and the expression of estrogen receptor (ER), progesterone receptor (PR) or human epidermal growth factor receptor 2 (Her2/neu). While hormone-based pharmacotherapies directly target these receptors (1-3), triple-negative breast cancer (TNBC) is more likely to recur earlier at distant sites, thus resulting in poor overall prognoses (4-7), due to the absence of these targets $(4,5,7)$. Notably, $\sim 15 \%$ of breast cancer cases are diagnosed as TNBC (8-10). However, successful therapies for the treatment of TNBC are currently unavailable. Therefore, novel therapeutic strategies are required for TNBC treatment.

Previous studies have reported that histone deacetylase inhibitors (HDACis) may be considered a promising novel class of anticancer agents $(11,12)$. HDACis are able to induce numerous cellular epigenetic alterations, enhance acetylation of various proteins, including transcription factors, molecular chaperones and structural components $(11,13,14)$, and modulate the growth, differentiation and survival of cells (15-18). Notably, it has been revealed that HDACis serve a critical role in modulating cell cycle arrest, apoptosis, angiogenesis, oncogene expression, and tumor cell invasion and metastasis $(11,12,19,20)$. However, the exact mechanism underlying the anticancer effects of HDACis remains unclear.

Previous studies have demonstrated that HDACis suppress breast cancer cells in vitro and in vivo. The effects of HDACis on breast cancer cells may be due to their ability to alter the expression of hormone receptors, including ER and PR. Specifically, previous studies have reported that HDACis can increase ER and PR expression levels $(21,22)$. In addition, HDACis can induce apoptosis and autophagy of breast cancer cells exhibiting Her-2 overexpression and basal-like/TNBC, and may reduce Her-2 gene expression. While previous studies have indicated that microRNAs (miRNAs/miRs) serve an important role in the effects of HDACis on Her-2 gene expression, the mechanisms underlying the effects of HDACis on ER and PR expression remain unknown (23-25). Therefore, the present study aimed 
to examine the role of miRNAs in the effects of HDAC is on breast cancer cells.

\section{Materials and methods}

Cell culture. The human breast cancer cell lines MDA-MB-231 and BT474 were obtained from the American Type Culture Collection (Manassas, VA, USA). All cells were maintained in Dulbecco's modified Eagles's medium/F-12 (1:1) medium (Invitrogen; Thermo Fisher Scientific, Inc., Waltham, MA, USA) supplemented with $10 \%$ fetal bovine serum (Invitrogen; Thermo Fisher Scientific, Inc.), and were cultured at $37^{\circ} \mathrm{C}$ in a humidified atmosphere containing $95 \%$ air and $5 \% \mathrm{CO}_{2}$. The cells were split twice a week.

miRNA expression microarray. Total RNA was isolated from cells using TRIzol ${ }^{\circledR}$ (Invitrogen; Thermo Fisher Scientific, Inc.). An ND-1000 spectrophotometer (NanoDrop Technologies; Thermo Fisher Scientific, Inc., Wilmington, DE, USA) with an Experion system (Bio-Rad Laboratories, Inc., Hercules, CA, USA) was used to quantify and confirm the quality of RNA samples. Extracted total RNA was labeled with Hy5 using the miRCURY LNA ${ }^{\mathrm{TM}}$ microRNA Hy5 Power Labeling kit (Exiqon, Inc., Woburn, MA, USA). Labeled RNAs were hybridized onto 3D-Gene Human miRNA Oligo chips (v.14 1.0.1; Toray Industries, Inc., Tokyo, Japan). The annotation and oligonucleotide sequences of the probes were confirmed using the miRBase miRNA database release 14 (http:// www.mirbase.org/). After numerous washes with 3D-Gene Human miRNA Oligo chips, fluorescent signals were scanned using the ScanArray Lite Scanner (Perkin-Elmer, Inc., Waltham, MA, USA) and were analyzed with GenePix Pro 7 software (Molecular Devices, LLC, Sunnyvale, CA, USA). Raw data were normalized by subtracting the mean intensity of the background signal (blank spots). Signal intensities $>2$ standard deviations of the background signal intensity were considered to be valid. The relative expression levels of a given miRNA were calculated by comparing the signal intensities of the averaged valid spots with their mean value throughout the microarray experiments. The data were normalized, and the significantly differentially expressed miRNAs were obtained by comparing cMCL with aMCL using an independent sample t-test $(\mathrm{P}<0.05)$. A heat map of expression data from the selected miRNAs was generated using MeV software (http://mev.tm4.org/).

Reverse transcription-polymerase chain reaction $(R T)-P C R$ and $R T$-quantitative $(q) P C R$. Total RNA was extracted using a modified chloroform/phenol procedure (TRIzol ${ }^{\circledR}$; Invitrogen; Thermo Fisher Scientific, Inc.). First-strand cDNA was generated using a High Capacity cDNA Reverse Transcription kit (Applied Biosystems; Thermo Fisher Scientific, Inc.) according to the manufacturer's protocol. To quantify mRNA levels, RT-qPCR was performed using ABsolute Blue qPCR Master Mix (Thermo Fisher Scientific, Inc.) according to the manufacturer's protocol. The primers used were as follows: ER $\alpha$ forward, 5'-TGATGAAAGGTGGGATACGA-3' and reverse, 5'-AAGGTTGGCAGCTCTCATGT-3'; PR forward, 5'-TCGAGTCATTACCTCAGAAGAT-3' and reverse, 5'-CCCACAGGTAAGGACACCATA-3'; HER2 forward, 5'-AACTGCACCCACTCCTGTGT-3' and reverse,
5'-TGATGAGGATCCCAAAGACC-3'; $\beta$-actin forward, 5'-CTGTGGCATCCACGAAACTA-3' and reverse, 5'CGCTCAGGAGGAGCAATG-3'. $\beta$-actin expression was used as an internal control for conventional RT-PCR and RT-qPCR. The thermocycling conditions were as follows: RT-PCR, $1 \mu \mathrm{g}$ total RNA was reverse-transcribed using the GeneAmp RNA PCR kit (Perkin-Elmer, Inc.). Subsequently, $2 \mu \mathrm{l}$ of the resulting cDNA samples was used in each PCR. The routine PCR program was 30 cycles of $1 \mathrm{~min}$ at $94^{\circ} \mathrm{C}, 1 \mathrm{~min}$ at $60^{\circ} \mathrm{C}$ (annealing temperature), and $1 \mathrm{~min}$ at $72^{\circ} \mathrm{C}$; RT-qPCR, first-strand cDNA was generated using a High Capacity cDNA Reverse Transcription kit (Applied Biosystems; Thermo Fisher Scientific, Inc.) according to the manufacturer's protocol. To quantify mRNA levels, RT-qPCR was performed using ABsolute Blue qPCR Master Mix (Thermo Fisher Scientific, Inc.) according to the manufacturer's protocol. All RT-qPCR reactions were conducted using a 7500 Fast Real-Time PCR system (Applied Biosystems; Thermo Fisher Scientific, Inc.) under the following conditions: $95^{\circ} \mathrm{C}$ for $3 \mathrm{~min}$, followed by 40 cycles of $15 \mathrm{sec}$ at $95^{\circ} \mathrm{C}$ and $30 \mathrm{sec}$ at $55^{\circ} \mathrm{C}$. All RT-qPCR reactions were conducted using a 7500 Fast Real-Time PCR system (Applied Biosystems; Thermo Fisher Scientific, Inc.). For RT-PCR, after PCR, the products were resolved in $2 \%$ agarose gel with ethidium bromide. The images were captured under UV transillumination. For RT-qPCR, the mRNA expression level was quantified by determining the cycle threshold $\left(\mathrm{C}_{\mathrm{T}}\right)$, which is the number of PCR cycles required for the fluorescence to exceed a value significantly higher than the background fluorescence.

Analysis of miRNA expression. Total RNA, including small RNA, was extracted and purified using the miRNeasy Mini kit (Qiagen, Inc., Valencia, CA, USA) according to the manufacturer's protocol. TaqMan MicroRNA RT kit (Applied Biosystems; Thermo Fisher Scientific, Inc.) was first used to generate cDNA using hairpin primers. The expression levels of specific miRNAs were measured by qPCR using TaqMan MicroRNA Assays, according to the manufacturer's protocol. RNU6B was used as an internal control to normalize all data using the TaqMan RNU6B assay (Applied Biosystems; Thermo Fisher Scientific, Inc.). RNU6B levels were unaffected by HDACis treatment. The relative miRNA expression levels were calculated using the comparative Cq method ( $\Delta \Delta \mathrm{Cq})(26)$.

Transfection of cells with miRNA inhibitors. Cell transfection with miRNA hairpin inhibitors or negative control inhibitors was conducted using HiPerFect Transfection Reagent (Qiagen, Inc.) according to the manufacturer's protocol. The miRIDIAN hairpin inhibitors of miR-762 or miR-642a-3p and the corresponding negative control (NC) were purchased from Thermo Scientific Dharmacon (Shanghai, China). BT474 cells were seeded into 96-well plates at a density of $2 \times 10^{5}$ cells/well and cultured at $37^{\circ} \mathrm{C}$ for $24 \mathrm{~h}$. A final concentration of $10 \mathrm{nM}$ miR-762 or miR-642a-3p or NC was transfected into target cells using Hiperfect (Qiagen, Inc.) and Opti-MEM ${ }^{\circledR}$ I reduced serum medium (Thermo Fisher Scientific, Inc.), according to the manufacturer's protocol.

Cell proliferation assay and quantification of apoptosis. Cell viability was determined using the CellTiter $96^{\circledR}$ AQueous Non-Radioactive Cell Proliferation Assay kit (Thermo Fisher 
Scientific, Inc.). Briefly, cells $\left(2 \times 10^{5}\right.$ cells/well) were plated into 96-well plates with complete medium for $24 \mathrm{~h}$, and were cultured in control medium or medium containing a series of doses of HDACis for a further $48 \mathrm{~h}$ at $37^{\circ} \mathrm{C}$. In addition, a specific apoptotic ELISA kit (cat. no. 11544675001; Roche Diagnostics, Indianapolis, IN, USA) was used to quantitatively measure cytoplasmic histone-associated DNA fragments (mononucleosomes and oligonucleosomes) according to the manufacturer's protocol.

Western blot analysis. Protein expression levels were determined by western blot analysis. Cells were lysed in RIPA buffer $(50 \mathrm{mM}$ Tris- $\mathrm{HCl}, \mathrm{pH} 7.4,150 \mathrm{mM} \mathrm{NaCl}, 1 \%$ Triton $\mathrm{X}-100,1 \%$ sodium dodecyl sulfate, $1 \%$ sodium deoxycholate, $1 \mathrm{mM}$ EDTA) together with a complete (EDTA-free) protease inhibitor cocktail (Kodak, Rochester, NY, USA), $1 \mathrm{mM}$ phenylmethylsulfonyl fluoride, and phosphatase inhibitors (5 mM sodium orthovanadate). Protein concentration was determined using Bradford Reagent (Bio-Rad Laboratories, Inc.). Equal amounts of total cell lysates were boiled in Laemmli SDS-sample buffer. Samples containing equal amounts of protein $(50 \mu \mathrm{g})$ per lane were loaded and separated by SDS-PAGE in 10\% gels and transferred to nitrocellulose membranes (Bio-Rad Laboratories, Inc.). The membranes were incubated for $1 \mathrm{~h}$ in $5 \%$ skimmed milk and probed overnight with specific primary antibodies (mouse monoclonal anti- $\beta$-actin (A5316; dilution 1:1,000; Sigma-Aldrich, Shanghai, China), rabbit monoclonal anti-ER $\alpha$ (ab108398; dilution 1:1,000), rabbit monoclonal anti-HER2/ ErbB2 (ab134182; dilution 1:1,000), and rabbit polyclonal anti-progesterone receptor (ab32085; dilution 1:1,000) (all from Abcam, Shanghai, China) at $4^{\circ} \mathrm{C}$. Subsequently, the blots were incubated with horseradish peroxidase-labeled secondary goat anti-rabbit antibody (Sigma-Aldrich). Finally, the signals were detected using enhanced chemiluminescence reagents (GE Healthcare Bio-Sciences, Pittsburgh, PA, USA).

Statistical analysis. All experiments were repeated at least two times. Data are presented as means \pm SD. Statistical analyses of the experimental data were determined using one-way analysis of variance followed by Bonferroni correction, where appropriate. Statistical analysis was performed using SPSS version 11.0 statistic software package. $\mathrm{P}<0.05$ was considered to indicate a statistically significant difference.

\section{Results}

Effects of HDACis on the mRNA expression levels of ER, PR and Her2/neu in breast cancer cells. In order to examine the effects of HDACis on the mRNA expression levels of ER, PR and Her2/neu in breast cancer cells, MDA-MB-231 and BT474 cells were treated with trichostatin A (TSA; 0.1 or $0.2 \mu \mathrm{M}$ ) or vorinostat (SAHA; 1.0 or $5.0 \mu \mathrm{M}$ ) for $48 \mathrm{~h}$ (Fig. 1). Conventional RT-PCR and RT-qPCR revealed that treatment with TSA or SAHA for $48 \mathrm{~h}$ dose-dependently enhanced the mRNA expression levels of ER and PR in MDA-MB-231 and BT474 cells (Fig. 1A, B, D and E). Furthermore, treatment with TSA or SAHA for $48 \mathrm{~h}$ dose-dependently reduced the mRNA expression levels of Her2/neu in MDA-MB-231 and BT474 cells (Fig. 1C and F). These findings suggested that
TSA and SAHA may alter ER, PR and Her2/neu expression via a transcription-dependent mechanism.

TSA and SAHA enhance the protein expression levels of ER and $P R$, and reduce the protein expression levels of Her2/neu. The present study also investigated whether TSA or SAHA may alter ER, PR and Her2/neu expression at the protein level. TSA and SAHA increased the protein expression levels of ER and PR in MDA-MB-231 and BT474 cells (Fig. 2). In addition, TSA and SAHA dose-dependently reduced the protein expression levels of Her2/neu in BT474 cells, which exhibit Her2/neu overexpression, but not in MDA-MB-231 cells, which is a TNBC cell line (Fig. 2).

TSA and SAHA treatment alters miRNA expression levels in BT474 cells. It is well known that miRNAs generally regulate gene expression by targeting mRNAs for degradation or translational repression (27-29). In addition, it has been reported that HDAC inhibition leads to rapid alteration of miRNA expression (30). In the present study, the mRNA and protein expression levels of ER, PR and Her2/neu were markedly altered in Her2/neu-overexpressing BT474 cells upon TSA or SAHA treatment. Therefore, it may be hypothesized that TSA or SAHA induces the expression of specific miRNAs that target ER, PR and Her2/neu mRNA. In order to explore the putative miRNAs that may be involved in modulation of ER, PR and Her2/neu mRNA and protein expression in BT474 cells, miRNA expression profiling was conducted using microarrays, after BT474 cells were treated with TSA $(0.2 \mu \mathrm{M})$ or SAHA $(5.0 \mu \mathrm{M})$ for $48 \mathrm{~h}$. A representative heat map was subsequently generated, as shown in Fig. 3. In general, treatment with TSA $(0.2 \mu \mathrm{M})$ and SAHA $(5.0 \mu \mathrm{M})$ induced marked alterations in the expression levels of various miRNAs in BT474 cells. The specific miRNAs are listed in Tables I-III.

SAHA downregulates Her2/neu expression and induces apoptosis of BT474 cells via miR-762 and miR-642a-3p. The present study aimed to determine whether the induction of specific miRNAs may have a causal role in downregulation of Her2/neu and apoptosis. Treatment with SAHA $(5.0 \mu \mathrm{M})$ for $48 \mathrm{~h}$ reduced Her $2 /$ neu mRNA expression and markedly reduced the protein expression levels of Her2/neu in BT474 cells. In addition, SAHA increased the expression levels of miR-762 and miR-642a-3p. Therefore, it was hypothesized that miR-762 and miR-642a-3p may serve a critical role in the effects of SAHA on Her2/neu expression and apoptosis of BT474 cells.

Initially, the present study confirmed that treatment with SAHA $(5.0 \mu \mathrm{M})$ for $48 \mathrm{~h}$ increased the expression levels of miR-762 and miR-642a-3p in BT474 cells, as determined using RT-qPCR (Fig. 4A). Specific miRIDIAN hairpin inhibitors were then transfected into BT474 cells to inhibit miR-762 or miR-642a-3p expression, the cells were then treated with or without SAHA $(5.0 \mu \mathrm{M})$. The results indicated that miR-762 and miR-642a-3p inhibitors significantly decreased SAHA-induced upregulation of miR-762 and miR-642a-3p, respectively (Fig. 4B). Notably, neither of the single inhibitors altered SAHA-induced downregulation of Her2/neu in BT474 cells (Fig. 4C). Furthermore, ELISA and western blot analyses were used to detect apoptosis; the results demonstrated that the 

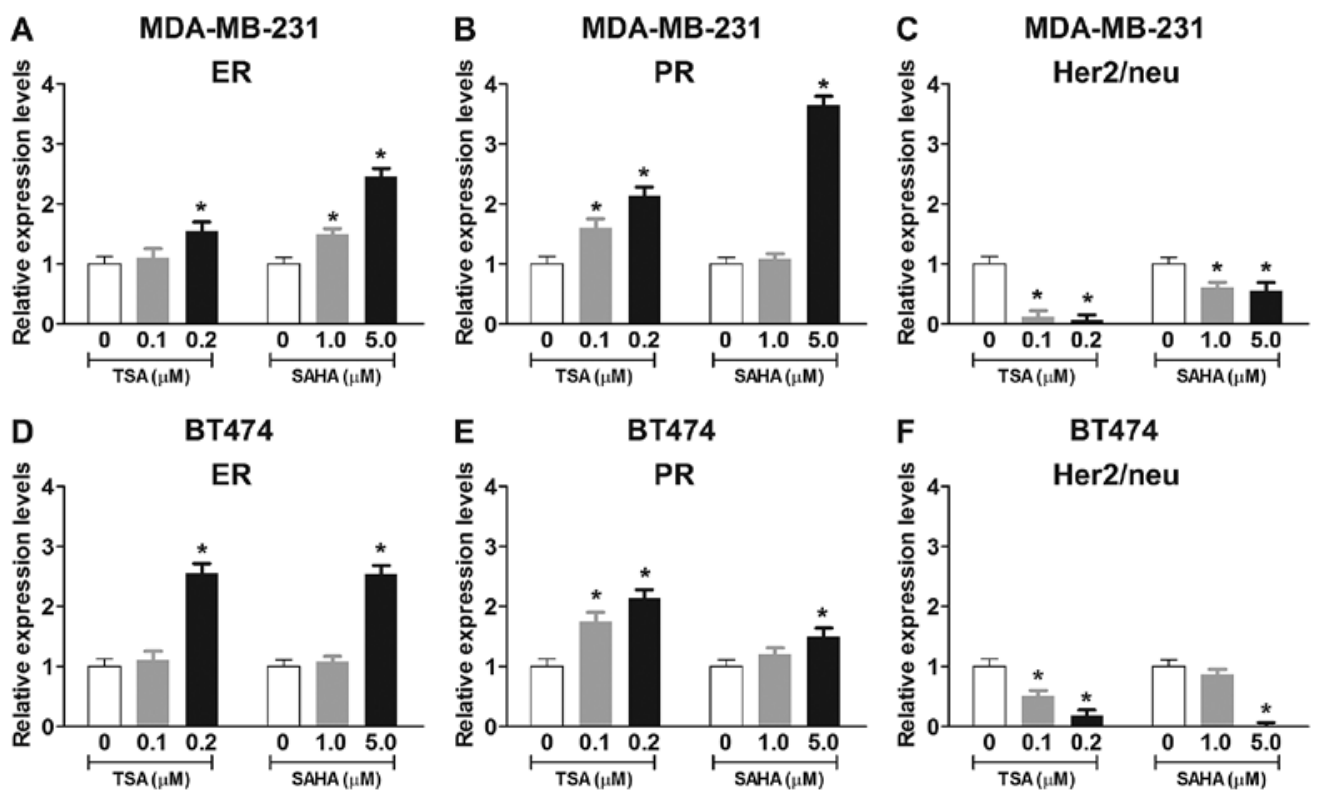

Figure 1. Treatment with TSA or SAHA affects the mRNA expression levels of ER, PR and Her2/neu in breast cancer cells. (A-C) MDA-MB-231 and (D-F) BT474 cells were treated with or without TSA and SAHA at the indicated concentrations for $48 \mathrm{~h}$. Cells were then subjected to total RNA extraction, and the mRNA expression levels of ER, PR, Her2/neu and $\beta$-actin were measured by reverse transcription-quantitative polymerase chain reaction. Data are representative of three independent experiments. "P<0.05 compared with vehicle $(0 \mu \mathrm{M})$ control. ER, estrogen receptor; Her $2 /$ neu, human epidermal growth factor receptor 2; PR, progesterone receptor; SAHA, vorinostat; TSA, trichostatin A.
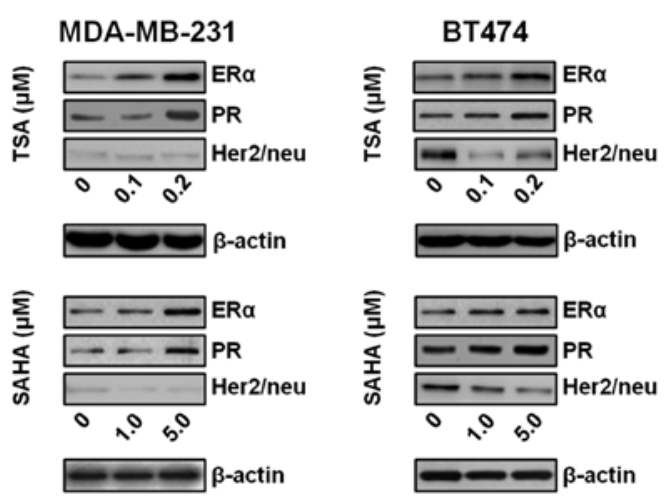

Figure 2. TSA and SAHA reduce the protein expression levels of ER, PR and Her2/neu in breast cancer cells. MDA-MB-231 and BT474 cells were treated with or without TSA and SAHA at the indicated concentrations for $48 \mathrm{~h}$. Proteins were then collected and subjected to western blot analyses with specific antibodies directed against ER, PR, Her2/neu and $\beta$-actin. ER, estrogen receptor; Her2/neu, human epidermal growth factor receptor 2; PR, progesterone receptor; SAHA, vorinostat; TSA, trichostatin A.

single miRNA inhibitors did not alter SAHA-induced DNA fragmentation and poly (ADP-ribose) polymerase (PARP) cleavage, which are hallmarks of apoptosis (Fig. 4D). These results suggested that inhibition of a single miRNA may be insufficient to attenuate the effects of SAHA on Her2/neu expression and cell apoptosis.

It has previously been suggested that numerous miRNAs work cooperatively to reduce the protein expression levels of Her2/neu (25); therefore, the present study cotransfected BT474 cells with the two miRNA inhibitors. Similarly, cotransfection with the two specific inhibitors markedly reduced expression of their corresponding miRNAs in BT474 cells (data not shown). In addition, cotransfection with miR-762 and miR-642a-3p inhibitors markedly inhibited SAHA-induced downregulation
Table I. Effects of trichostatin $(0.2 \mu \mathrm{M})$ on miRNA expression in BT474 cells.

\begin{tabular}{lcc}
\hline miRNA & Fold-change & P-value \\
\hline miR-10a-5p & $\uparrow 8.41$ & 0.04 \\
miR-1587 & $\uparrow 1.24$ & 0.03 \\
miR-4634 & $\uparrow 1.47$ & 0.03 \\
miR-4687 & $\uparrow 1.17$ & 0.04 \\
miR-17-5p & $\downarrow 1.21$ & 0.04 \\
miR-18b-5p & $\downarrow 1.60$ & 0.04 \\
miR-20a-5p & $\downarrow 1.31$ & 0.01 \\
miR-20b-5p & $\downarrow 1.20$ & 0.003 \\
miR-301a-3p & $\downarrow 1.41$ & 0.03 \\
miR-5100 & $\downarrow 1.40$ & 0.03 \\
\hline
\end{tabular}

miRNA/miR, microRNA.

of Her2/neu in BT474 cells (Fig. 5A). Similarly, simultaneous inhibition of the two miRNAs reduced SAHA-induced apoptosis and PARP cleavage (Fig. 5B) in BT474 cells. These results indicated that numerous SAHA-induced miRNAs may be necessary to downregulate Her2/neu expression and promote apoptosis of Her2-overexpressing breast cancer cells.

\section{Discussion}

The present study demonstrated that TSA and SAHA dose-dependently enhanced the mRNA expression levels of ER and PR, and reduced Her2/neu mRNA expression in MDA-MB-231 and BT474 cells. Western blot analysis also confirmed that TSA and SAHA increased the protein expres- 


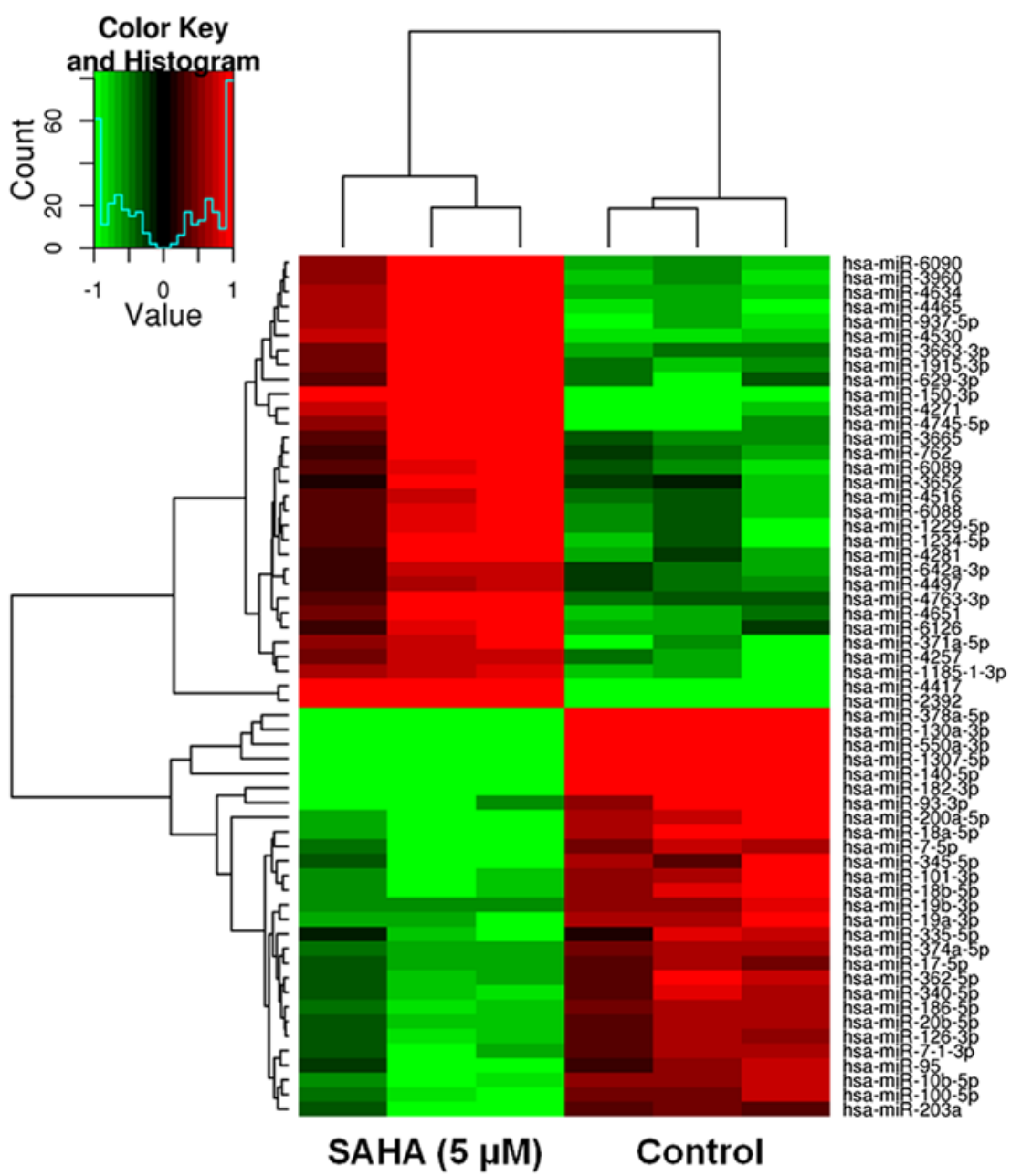

Figure 3. Representative heat map of miR microarray expression data. miR, microRNA; SAHA, vorinostat.

Table II. Effects of vorinostat $(5 \mu \mathrm{M})$ on miRNA expression in BT474 cells.

\begin{tabular}{lcl}
\hline miRNA & Fold-change & P-value \\
\hline miR-150-3p & $\uparrow 5.01$ & 0.0006 \\
miR-937-5p & $\uparrow 4.45$ & 0.004 \\
miR-629-3p & $\uparrow 4.48$ & 0.03 \\
miR-4634 & $\uparrow 3.63$ & 0.016 \\
miR-371a-5p & $\uparrow 3.02$ & 0.002 \\
miR-762 & $\uparrow 2.42$ & 0.03 \\
miR-642a-3p & $\uparrow 2.11$ & 0.008 \\
miR-18a-5p & $\downarrow 4.01$ & 0.000 \\
miR-200a-5p & $\downarrow 6.01$ & 0.04 \\
miR-10b-5p & $\downarrow 2.61$ & 0.001 \\
miR-18b-5p & $\downarrow 3.02$ & 0.01 \\
miR-19a-3p & $\downarrow 2.50$ & 0.001 \\
miR-19b-3p & $\downarrow 2.31$ & 0.004 \\
miR-20b-5p & $\downarrow 2.20$ & 0.002 \\
miR-17-5p & $\downarrow 2.02$ & 0.001 \\
miR-20a-5p & $\downarrow 1.80$ & 0.005 \\
miR-100b-5p & $\downarrow 2.50$ & 0.005 \\
\hline
\end{tabular}

miRNA/miR, microRNA.
Table III. Comparison of the effects of SAHA $(5 \mu \mathrm{M})$ and TSA $(0.2 \mu \mathrm{M})$ on miRNA expression levels in BT474 cells.

\begin{tabular}{lcc}
\hline miRNA & TSA (fold) & SAHA (fold) \\
\hline miR-4634 & $\uparrow 1.47$ & $\uparrow 3.63$ \\
miR-17-5p & $\downarrow 1.21$ & $\downarrow 2$ \\
miR-18b-5p & $\downarrow 1.60$ & $\downarrow 3$ \\
miR-20a-5p & $\downarrow 1.30$ & $\downarrow 1.8$ \\
miR-20b-5p & $\downarrow 1.20$ & $\downarrow 2.2$ \\
miR-301a-3p & $\downarrow 1.40$ & $\downarrow 1.6$ \\
miR-5100 & $\downarrow 1.41$ & $\downarrow 1.34$
\end{tabular}

miRNA/miR, microRNA; SAHA, vorinostat; TSA, trichostatin A.

sion levels of ER and PR in MDA-MB-231 and BT474 cells. In addition, TSA and SAHA dose-dependently reduced the protein expression levels of Her2/neu in the Her2/neu-overexpressing cell line, BT474, but not in the TNBC cell line, MDA-MB-231. It has previously been reported that HDAC inhibition leads to marked alterations in miRNA expression (30). Therefore, the present study explored the putative miRNAs that may be involved in the modulation of ER, PR and Her2/neu mRNA and 

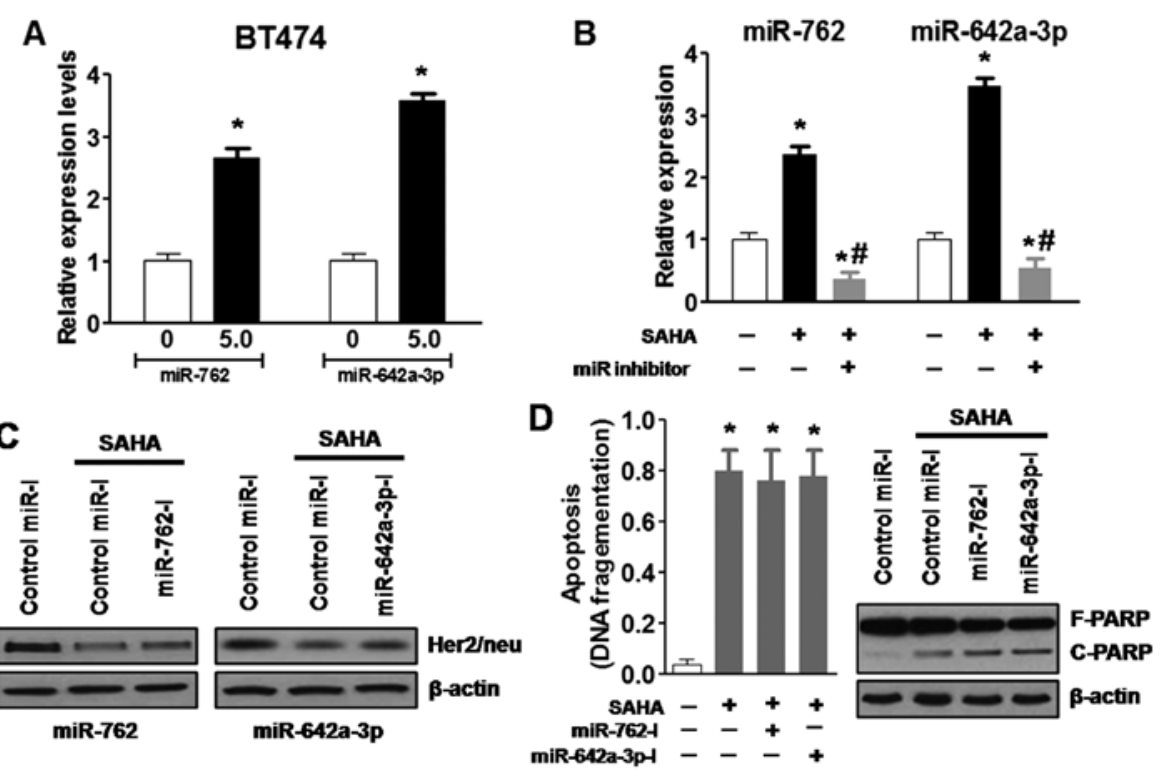

Figure 4. SAHA downregulates Her2/neu expression and induces apoptosis of BT474 cells, potentially via miR-762 and miR-642a-3p. (A) SAHA (5.0 $\mu$ M) treatment for $48 \mathrm{~h}$ increased miR-762 and miR-642a-3p expression in BT474 cells, as determined by reverse transcription-quantitative polymerase chain reaction. (B) miR-762 and miR-642a-3p inhibitors significantly decreased SAHA-induced upregulation of miR-762 and miR-642a-3p, respectively. (C) Single miRNA inhibitors did not alter SAHA-induced downregulation of Her2/neu in BT474 cells. (D) Single miRNA inhibitors did not alter SAHA-induced DNA fragmentation and PARP cleavage, which are hallmarks of apoptosis. " $\mathrm{P}<0.05$, compared with the control group; ${ }^{~} \mathrm{P}<0.05$, compared with the SAHA group. F-PARP, full-length PARP; C-PARP, cleaved-PARP; Her2/neu, human epidermal growth factor receptor 2; miR/miRNA, microRNA; PARP, poly (ADP-ribose) polymerase; SAHA, vorinostat.
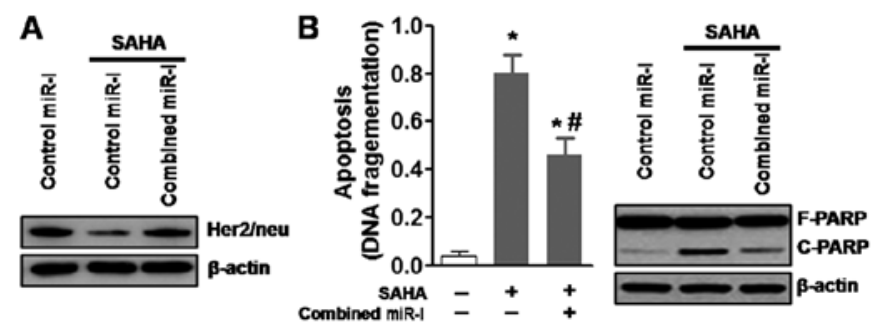

Figure 5. Cotransfection with miR-762 and miR-642a-3p inhibitors alters SAHA-induced downregulation of Her $2 /$ neu expression and SAHA-induced apoptosis of BT474 cells. (A) Cotransfection with miR-762 and miR-642a-3p inhibitors markedly inhibited SAHA-induced downregulation of Her2/neu in BT474 cells. (B) Simultaneous inhibition of two miRNAs reduced SAHA-induced apoptosis and PARP cleavage in BT474 cells. " $\mathrm{P}<0.05$, compared with the control group; ${ }^{~} \mathrm{P}<0.05$, compared with the SAHA group. F-PARP, full-length PARP; C-PARP, cleaved-PARP; Her2/neu, human epidermal growth factor receptor 2; miR/miRNA, microRNA; PARP, poly (ADP-ribose) polymerase; SAHA, vorinostat.

protein expression in BT474 cells. Briefly, miRNA expression profiling was conducted using microarrays after BT474 cells were treated with TSA $(0.2 \mu \mathrm{M})$ or SAHA $(5.0 \mu \mathrm{M})$ for $48 \mathrm{~h}$. The results indicated that TSA $(0.2 \mu \mathrm{M})$ and SAHA $(5.0 \mu \mathrm{M})$ induced a marked alteration in the expression levels of various miRNAs in BT474 cells. Subsequently, the present study aimed to determine whether induction of these miRNAs serves a causal role in the downregulation of Her2/neu and apoptosis. Focusing on miR-762 and miR-642a-3p, the present study confirmed that SAHA $(5.0 \mu \mathrm{M})$ treatment for $48 \mathrm{~h}$ increased miR-762 and miR-642a-3p expression in BT474 cells. Notably, compared with delivery of miR-762 and miR-642a-3p inhibitors alone, cotransfection with the inhibitors markedly inhibited SAHA-induced downregulation of Her2/neu, and significantly reduced SAHA-induced apoptosis and PARP cleavage in BT474 cells. These results indicated that numerous SAHA-induced miRNAs are required to downregulate Her2/neu and promote apoptosis of Her2-overexpressing breast cancer cells.

Notably, whereas the mRNA expression levels of Her2 were decreased in both MDA-MB-231 and BT474 cells following TSA or SAHA treatment, the protein expression levels of Her2 were not altered in MDA-MB-231 cells. Numerous processes exist between transcription and translation, which may contribute to this discrepancy. In addition, the correlation between mRNA and protein expression can be as little as $40 \%$, depending on the type of cells and tissues $(31,32)$. There are various regulatory processes that occur following mRNA production, including post-transcriptional and translational regulation, and protein degradation, which may have a critical role in controlling steady-state protein levels (31). In addition, the half-life of various proteins can vary from minutes to days, whereas the degradation rate of mRNA falls within a much tighter range. Furthermore, the mRNA transcription rate is usually lower compared with protein translation in mammalian cells. Therefore, reduced mRNA levels may not alter protein levels, since slower degradation and/or a higher translation rate may overcome the lower levels of mRNA. Finally, the MDA-MB-231 cell line is a Her2-negative cell line; therefore, the basal levels of Her2 protein are much lower. The negative effects of HDACis on Her2 protein expression may also be due to the basement effects.

A previous study has revealed the functional cooperation of miR-125a, miR-125b and miR-205 in entinostat-induced Her2/neu downregulation and apoptosis of breast cancer cells (25). The present study suggested that miR-762 and miR-642a-3p may have a critical role in the modulation of Her2/neu expression in Her2-overexpressing breast cancer cells. A previous study also demonstrated that SAHA can 
reduce the mRNA expression levels of Her2/neu (25). Consistent with these findings, in the present study, SAHA and TSA reduced the mRNA expression levels of Her2/neu in MDA-MB-231 and BT474 cells. Compared with the effects of entinostat on miRNA expression, the present microarray results indicated that TSA and SAHA did not alter the expression levels of miR-125a, miR-15b or miR-205 in BT474 cells. Therefore, these results suggested that specific HDACis may induce a unique profile of miRNAs, and certain HDACis may regulate Her2/neu expression via different miRNAs.

It has previously been demonstrated that miRNA clusters may function cooperatively to regulate specific signaling pathways (33). Furthermore, it has been confirmed that numerous miRNAs can target the same gene $(25,34)$. To the best of our knowledge, the present study is the first to demonstrate that miR-762 and miR-642a-3p act cooperatively to regulate the expression of Her $2 /$ neu in breast cancer cells. Compared with simultaneous inhibition of the two miRNAs, inactivation of one single miRNA was unable to inhibit SAHA-induced downregulation of Her2/neu. However, cotransfection with the two miRNA inhibitors did not exhibit full efficacy, thus suggesting that additional miRNAs may be involved in regulating SAHA-induced downregulation of Her2/neu. Future studies are requited to further elucidate the mechanisms.

The Her family consists of at least four members, including EGFR (Her1, erbB1), Her2 (erbB2, Her2/neu), Her3 (erbB3) and Her4 (erbB4) (35). These family members are often aberrantly activated in various types of cancer, particularly in breast cancer, and are excellent targets for selective anticancer therapies. In clinical treatment, erbB-targeted therapies usually consist of antibodies, including trastuzumab, which target erbB2, and tyrosine kinase inhibitors, such as lapatinib, which target EGFR and erbB2. In addition, erbB3-targeted therapies are currently being evaluated in preclinical studies $(36,37)$, and numerous anti-erbB3 antibodies may be considered promising therapies for cancer treatment (38). Notably, erbB2 and erbB3 functionally interact with each other. For example, it has been reported that erbB3 is required for erbB2 to promote breast cancer cell proliferation $(39,40)$. Furthermore, erbB3 is critically involved in erbB2-mediated tamoxifen and paclitaxel resistance $(41,42)$. Therefore, future studies may aim to examine the effects of TSA and SAHA, as well as other HDACis, on erbB3, since simultaneously targeting erbB2 and erbB3 may have a broader impact on the treatment of breast cancer.

The molecular mechanisms by which SAHA and TSA induce expression of various miRNAs in breast cancer cells remain unclear. Numerous studies have indicated that epigenetic alterations, including DNA methylation and histone modifications, are likely the major mechanisms underlying miRNA expression regulation. Both acetylated-histone $\mathrm{H} 3$ and methylated-histone $\mathrm{H} 3$ are associated with open chromatin structure and active gene, including miRNA, expression $(43,44)$. Previous studies have demonstrated that TSA and SAHA treatment may increase acetylation of histone $\mathrm{H} 3$ and induce candidate miRNA expression $(45,46)$. It has also been reported that entinostat enhances acetylated-histone $\mathrm{H} 3$ and reduces HDAC1 (47). In addition, HDACis induce degradation of DNA methyltransferase 1, which is an enzyme responsible for maintaining DNA methylation patterns in eukaryotic cells and breast cancer cells $(44,48)$. Therefore, it is possible that both increased acetylated-histone $\mathrm{H} 3$ and reduced promoter methylation contribute to TSA and SAHA-induced upregulation of miRNAs in breast cancer cells.

In conclusion, the present study demonstrated that TSA and SAHA dose-dependently enhanced the mRNA and protein expression levels of ER and PR in MDA-MB-231 and BT474 cells, reduced Her2/neu mRNA expression in MDA-MB-231 cells, and reduced Her2/neu mRNA and protein expression in BT474 cells. Furthermore, TSA and SAHA treatment induced a marked alteration in the expression levels of various miRNAs in BT474 cells. Notably, when miR-762 and miR-642a-3p inhibitors were delivered together, combined inhibition markedly suppressed SAHA-induced downregulation of Her2/neu, and significantly reduced SAHA-induced apoptosis and PARP cleavage in BT474 cells. These results indicated that numerous HDACi-induced miRNAs are required to downregulate Her2/neu and promote apoptosis of Her2-overexpressing breast cancer cells. These findings may help further understanding regarding the roles of miRNA networks in cancer biology.

\section{Acknowledgements}

Not applicable.

\section{Funding}

The present study was supported by grants from the National Science and Technology Pillar Program (grant no. 2015BAI12B15) and the National Natural Science Foundation of China (grant nos. 81302080 and 81472183). The funders had no role in study design, data collection and analysis, decision to publish or preparation of the manuscript.

\section{Availability of data and materials}

The datasets used and/or analyzed during the current study are available from the corresponding author on reasonable request.

\section{Authors' contributions}

YS conducted the experiments, analyzed and interpreted the data, and prepared the manuscript. YJ conducted the microarray experiment. WZ conducted RT-PCR and RT-qPCR. LZ conducted the western blot analysis. XX conducted the cell transfection and apoptosis experiments. ZT designed the study and prepared the manuscript. All authors read and approved the final manuscript.

\section{Ethics approval and consent to participate}

Not applicable.

\section{Consent for publication}

Not applicable.

\section{Competing interests}

The authors declare that they have no competing interests. 


\section{References}

1. Fernández Y, Cueva J, Palomo AG, Ramos M, de Juan A, Calvo L, García-Mata J, García-Teijido P, Peláez I and García-Estévez L: Novel therapeutic approaches to the treatment of metastatic breast cancer. Cancer Treat Rev 36: 33-42, 2010

2. Beaumont $\mathrm{T}$ and Leadbeater $\mathrm{M}$ : Treatment and care of patients with metastatic breast cancer. Nurs Stand 25: 49-56, 2011.

3. Watanabe T: Treatment of metastatic breast cancer patients: What is the standard care of the patients? Jpn J Clin Oncol 28: 355-356, 1998.

4. Schneider BP, Winer EP, Foulkes WD, Garber J, Perou CM, Richardson A, Sledge GW and Carey LA: Triple-negative breast cancer: Risk factors to potential targets. Clin Cancer Res 14: 8010-8018, 2008.

5. Dent R, Trudeau M, Pritchard KI, Hanna WM, Kahn HK, Sawka CA, Lickley LA, Rawlinson E, Sun P and Narod SA: Triple-negative breast cancer: clinical features and patterns of recurrence. Clin Cancer Res 13: 4429-4434, 2007.

6. De Laurentiis M, Cianniello D, Caputo R, Stanzione B, Arpino G, Cinieri S, Lorusso V and De Placido S: Treatment of triple negative breast cancer (TNBC): Current options and future perspectives. Cancer Treat Rev 36 (Suppl 3): S80-S86, 2010.

7. Chacón RD and Costanzo MV: Triple-negative breast cancer. Breast Cancer Res 12 (Suppl 2): S3, 2010.

8. Jemal A, Bray F, Center MM, Ferlay J, Ward E and Forman D: Global cancer statistics. CA Cancer J Clin 61: 69-90, 2011.

9. Anders CK and Carey LA: Biology, metastatic patterns, and treatment of patients with triple-negative breast cancer. Clin Breast Cancer 9 (Suppl 2): S73-S81, 2009.

10. Hudis CA and Gianni L: Triple-negative breast cancer: An unmet medical need. Oncologist 16 (Suppl 1): 1-11, 2011.

11. Drummond DC, Noble CO, Kirpotin DB, Guo Z, Scott GK and Benz CC: Clinical development of histone deacetylase inhibitors as anticancer agents. Annu Rev Pharmacol Toxicol 45: 495-528, 2005

12. Liu T, Kuljaca S, Tee A and Marshall GM: Histone deacetylase inhibitors: Multifunctional anticancer agents. Cancer Treat Rev 32: 157-165, 2006.

13. Kikuchi H, Barman HK, Nakayama M, Takami Y and Nakayama T: Participation of histones, histone modifying enzymes and histone chaperones in vertebrate cell functions. Subcell Biochem 40: 225-243, 2006.

14. Konstantinopoulos PA, Karamouzis MV and Papavassiliou AG: Focus on acetylation: The role of histone deacetylase inhibitors in cancer therapy and beyond. Expert Opin Investig Drugs 16: 569-571, 2007.

15. Johnstone RW: Histone-deacetylase inhibitors: Novel drugs for the treatment of cancer. Nat Rev Drug Discov 1: 287-299, 2002.

16. Vigushin DM and Coombes RC: Histone deacetylase inhibitors in cancer treatment. Anticancer Drugs 13: 1-13, 2002.

17. Richon VM and O'Brien JP: Histone deacetylase inhibitors: A new class of potential therapeutic agents for cancer treatment Clin Cancer Res 8: 662-664, 2002.

18. Lin HY, Chen CS, Lin SP, Weng JR and Chen CS: Targeting histone deacetylase in cancer therapy. Med Res Rev 26: 397-413, 2006.

19. Kim HJ and Bae SC: Histone deacetylase inhibitors: Molecular mechanisms of action and clinical trials as anticancer drugs. Am J Transl Res 3: 166-179, 2011.

20. Xu WS, Parmigiani RB and Marks PA: Histone deacetylase inhibitors: Molecular mechanisms of action. Oncogene 26 : 5541-5552, 2007.

21. Wang C, Fu M, Angeletti RH, Siconolfi-Baez L, Reutens AT, Albanese C, Lisanti MP, Katzenellenbogen BS, Kato S, Hopp T, et al: Direct acetylation of the estrogen receptor alpha hinge region by $\mathrm{p} 300$ regulates transactivation and hormone sensitivity. J Biol Chem 276: 18375-18383, 2001.

22. Gaughan L, Logan IR, Cook S, Neal DE and Robson CN: Tip60 and histone deacetylase 1 regulate androgen receptor activity through changes to the acetylation status of the receptor. J Biol Chem 277: 25904-25913, 2002.

23. Bouchalova K, Cizkova M, Cwiertka K, Trojanec R and Hajduch M: Triple negative breast cancer - current status and prospective targeted treatment based on Her1 (EGFR), TOP2A and C-MYC gene assessment. Biomed Pap Med Fac Univ Palacky Olomouc Czech Repub 153: 13-17, 2009.

24. Rao R, Balusu R, Fiskus W, Mudunuru U, Venkannagari S, Chauhan L, Smith JE, Hembruff SL, Ha K, Atadja P, et al: Combination of pan-histone deacetylase inhibitor and autophagy inhibitor exerts superior efficacy against triple-negative human breast cancer cells. Mol Cancer Ther 11: 973-983, 2012.
25. Wang S, Huang J, Lyu H, Lee CK, Tan J, Wang J and Liu B Functional cooperation of miR-125a, miR-125b, and miR-205 in entinostat-induced downregulation of erbB2/erbB3 and apoptosis in breast cancer cells. Cell Death Dis 4: e556, 2013.

26. Livak KJ and Schmittgen TD: Analysis of relative gene expression data using real-time quantitative PCR and the 2(-Delta Delta C(T)) Method. Methods 25: 402-408, 2001.

27. Bartels CL and Tsongalis GJ: MicroRNAs: Novel biomarkers for human cancer. Clin Chem 55: 623-631, 2009.

28. Bartel DP: MicroRNAs: Target recognition and regulatory functions. Cell 136: 215-233, 2009

29. Friedman RC, Farh KK, Burge CB and Bartel DP: Most mammalian mRNAs are conserved targets of microRNAs. Genome Res 19: 92-105, 2009.

30. Scott GK, Mattie MD, Berger CE, Benz SC and Benz CC: Rapid alteration of microRNA levels by histone deacetylase inhibition. Cancer Res 66: 1277-1281, 2006.

31. Vogel C and Marcotte EM: Insights into the regulation of protein abundance from proteomic and transcriptomic analyses. Nat Rev Genet 13: 227-232, 2012.

32. Maier T, Güell $M$ and Serrano L: Correlation of mRNA and protein in complex biological samples. FEBS Lett 583: 3966-3973, 2009.

33. Mestdagh P, Boström AK, Impens F, Fredlund E, Van Peer G, De Antonellis P, von Stedingk K, Ghesquière B, Schulte $S$, Dews M, et al: The miR-17-92 microRNA cluster regulates multiple components of the TGF- $\beta$ pathway in neuroblastoma. Mol Cell 40: 762-773, 2010.

34. Wu S, Huang S, Ding J, Zhao Y, Liang L, Liu T, Zhan R and He X: Multiple microRNAs modulate p21Cip1/Waf1 expression by directly targeting its 3 ' untranslated region. Oncogene 29: 2302-2308, 2010.

35. Moasser MM: The oncogene Her2: Its signaling and transforming functions and its role in human cancer pathogenesis. Oncogene 26: 6469-6487, 2007.

36. Schoeberl B, Faber AC, Li D, Liang MC, Crosby K, Onsum M, Burenkova O, Pace E, Walton Z, Nie L, et al: An ErbB3 antibody, MM-121, is active in cancers with ligand-dependent activation. Cancer Res 70: 2485-2494, 2010.

37. Schoeberl B, Pace EA, Fitzgerald JB, Harms BD, Xu L, Nie L, Linggi B, Kalra A, Paragas V, Bukhalid R, et al: Therapeutically targeting ErbB3: A key node in ligand-induced activation of the ErbB receptor-PI3K axis. Sci Signal 2: ra31, 2009.

38. Aurisicchio L, Marra E, Roscilli G, Mancini R and Ciliberto G: The promise of anti-ErbB3 monoclonals as new cancer therapeutics. Oncotarget 3: 744-758, 2012.

39. Holbro T, Beerli RR, Maurer F, Koziczak M, Barbas CF III and Hynes NE: The ErbB2/ErbB3 heterodimer functions as an oncogenic unit: ErbB2 requires ErbB3 to drive breast tumor cell proliferation. Proc Natl Acad Sci USA 100: 8933-8938, 2003.

40. Lee-Hoeflich ST, Crocker L, Yao E, Pham T, Munroe X, Hoeflich KP, Sliwkowski MX and Stern HM: A central role for Her3 in Her2-amplified breast cancer: Implications for targeted therapy. Cancer Res 68: 5878-5887, 2008.

41. Liu B, Ordonez-Ercan D, Fan Z, Edgerton SM, Yang X and Thor AD: Downregulation of erbB3 abrogates erbB2-mediated tamoxifen resistance in breast cancer cells. Int J Cancer 120: 1874-1882, 2007

42. Wang S, Huang X, Lee CK and Liu B: Elevated expression of erbB3 confers paclitaxel resistance in erbB2-overexpressing breast cancer cells via upregulation of Survivin. Oncogene 29: 4225-4236, 2010.

43. Chuang JC and Jones PA: Epigenetics and microRNAs. Pediatr Res 61: 24R-29R, 2007.

44. Veeck J and Esteller M: Breast cancer epigenetics: From DNA methylation to microRNAs. J Mammary Gland Biol Neoplasia 1: 5-17, 2010.

45. Su X, Qian C, Zhang Q, Hou J, Gu Y, Han Y, Chen Y, Jiang M and Cao X: miNomes of haematopoietic stem cells and dendritic cells identify miR-30b as a regulator of Notch1. Nat Commun 4: 2903, 2013

46. Drogaris P, Villeneuve V, Pomiès C, Lee EH, Bourdeau V, Bonneil E, Ferbeyre G, Verreault A and Thibault P: Histone deacetylase inhibitors globally enhance $\mathrm{h} 3 / \mathrm{h} 4$ tail acetylation without affecting h3 lysine 56 acetylation. Sci Rep 2: 220, 2012.

47. Huang X, Gao L, Wang S, Lee CK, Ordentlich P and Liu B: HDAC inhibitor SNDX-275 induces apoptosis in erbB2-overexpressing breast cancer cells via down-regulation of erbB3 expression. Cancer Res 69: 8403-8411, 2009.

48. Zhou Q, Agoston AT, Atadja P, Nelson WG and Davidson NE: Inhibition of histone deacetylases promotes ubiquitin-dependent proteasomal degradation of DNA methyltransferase 1 in human breast cancer cells. Mol Cancer Res 6: 873-883, 2008. 